\title{
PREDICTION OF KNEE LOADS USING A LOWER EXTREMITY MODEL BASED ON THE KLEIN HORSMAN DATA SET
}

\author{
Cédric Schwartz ${ }^{1}$, Morten Enemark Lund ${ }^{2}$, Mark de Zee ${ }^{1}$, John Rasmussen ${ }^{2}$ \\ ${ }^{1}$ Department of Health Science and Technology \\ Aalborg University \\ Aalborg \\ Denmark \\ ${ }^{2}$ Department of Mechanical Engineering \\ Aalborg University \\ Aalborg \\ Denmark
}

\section{INTRODUCTION}

In this paper, the in-vivo loads of the knee joint provided by an instrumented prosthesis (Fregly et al., 2010, Lin et al., 2010, Kim et al., 2009) are compared to the results obtained from an implementation of the Klein Horsman data set (2007) in the AnyBody Modeling System. The lateral and medial knee contact forces are estimated directly from the knee modeled as a modified revolute joint. As such, this study presents what can be achieved by estimating the knee contact forces from a simplified knee model.

\section{MATERIALS AND METHODS}

This study was a part of the "Grand Challenge Competition to Predict In-Vivo Knee Loads" during the 2010 Summer Bioengineering Conference. The study is based on the synchronized data set provided for the competition.

\section{Experimental in-vivo data}

Data was collected from one patient (age: 83, height: $1.66 \mathrm{~m}$, mass: $64.6 \mathrm{~kg}$ ) implanted with an instrumented knee replacement in the right knee. The subject performed a set of calibration trials and gait trials (normal gait and medial-lateral trunk sway gait). The competition data set provided a large set of experimental data, but only the following was used to create the model: tibial contact forces (Zhao et al., 2007), ground reaction forces and marker trajectories. The subject motion was acquired by measuring 3D position of 31 skin markers placed in a modified Cleveland Clinic Marker set. Four force plates gave access to the ground reaction forces during motion.

\section{Biomechanical model used for loads estimation}

A three-dimensional musculoskeletal model of the body (Figure 1) based on the Klein Horsman data set (2007) was built using the AnyBody Modeling System (Damsgaard et al., 2006). This leg model is a 6 degree-of-freedom (DOF) model actuated by 55 muscles divided into 159 fascicles per leg. The knee joint was defined as a 1 DOF joint. Flexion/extention rotation was driven by the subject specific motion. The knee also includes posterior-anterior translation given by the equation provided in Walker et al., 1988. In this study, only the linear term was kept. Musculotendinous units were modeled as isometric strength actuators. Because of small joint angle variations and moderate contraction velocities during the studied gait trials, this simple approach is still relevant. The musculoskeletal model was scaled to the specific size of the subject (Rasmussen et al., 2005). The muscle recruitment was then performed by a so-called min-max criterion, which minimizes the maximum muscle activity (Rasmussen al., 2001).

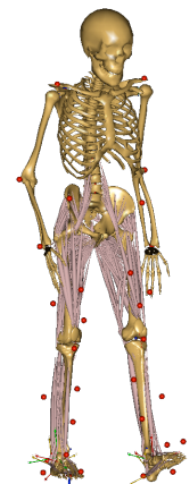

Figure 1: Lower extremity model based on the Klein Horsman data set (scaled to the studied subject)

In the default knee revolute joint, the reaction forces and moments correspond directly to the non-physiological mechanical joint constraints at the knee center. For our purpose, the reaction force in tibia long axis direction as well as the inward-outward reaction moment were removed and replaced by two "contact" elements representing the two femoral condyles. The distance between the 2 "contact" points is equal to $40 \mathrm{~mm}$, which is also the distance between the sensors of the instrumented prosthesis. The line of action of the two contacts was collinear to the long axis of the tibia and the elements were only able to "push". The medial and lateral loads measurements were realized on these two "contact" points located on the knee rotation axis and were estimated as the force needed by these two elements to ensure the joint coherence.

\section{RESULTS}

Figure 2 compares the loads predicted by the model and the invivo loads measured by the prosthesis during a squat movement. In neutral position both measures of total loads (lateral plus medial) are equal to 0.5 BW (Body Weight). For maximal flexion $\left(90^{\circ}\right)$, the predicted loads are respectively in the medial and lateral compartment of $810 \mathrm{~N}$ and $1550 \mathrm{~N}$. The corresponding results are $670 \mathrm{~N}$ and $750 \mathrm{~N}$.

Figure 3 presents the loads estimated by AnyBody modeling system on five normal gait trials and five trunk sway trials. In all trials, maximum predicted loads during the stance phase occurred in the beginning of the stance phase and during propulsion. Maximal loads 
are approximately equal to $1500 \mathrm{~N}$ in the medial and $1100 \mathrm{~N}$ in the lateral compartment. The trunk sway gait presented the same pattern as the normal gait but with higher loads in both compartments in the beginning of the stance phase compared to normal gait. Maximal loads are now approximately equal to $1550 \mathrm{~N}$ in the medial compartment and $1500 \mathrm{~N}$ in the lateral one. It can also be noticed that the repeatability of this motion was not as good as during the normal trial.
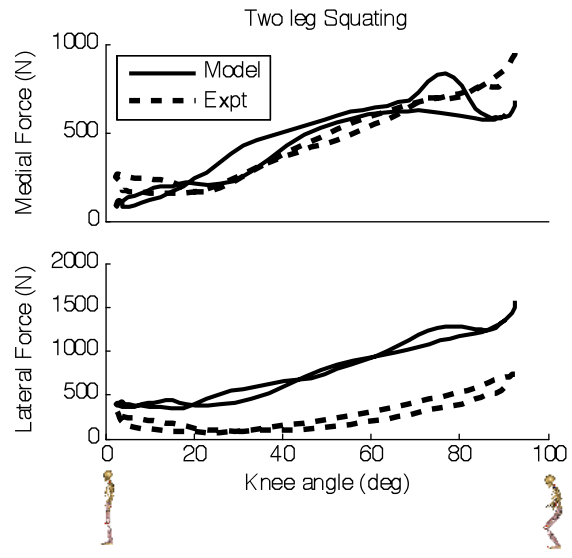

Figure 2: Knee loads (medial and lateral plateau) predicted with AnyBody and the in-vivo loads measured by the instrumented prosthesis during a squat trial.

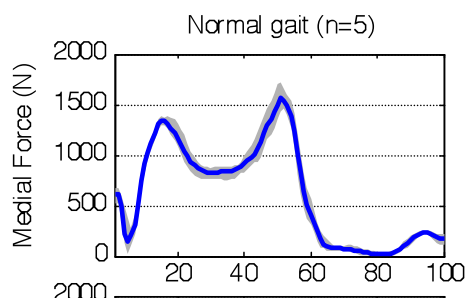

Trunk sway gait $(n=5)$
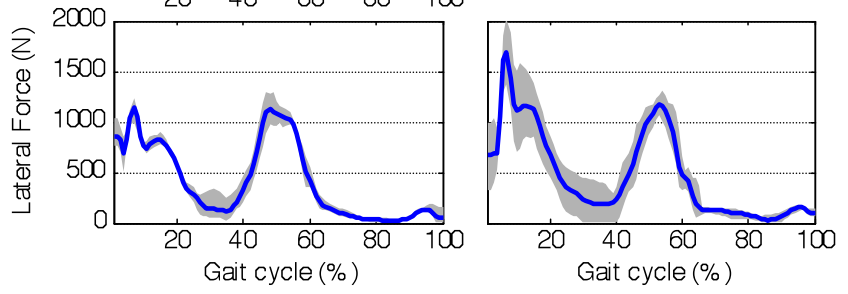

Figure 3: Mean (blue) and range (grey area) knee loads predicted for five normal and five trunk sway gait trials.

\section{DISCUSSION}

The knee joint model used in this study to predict the medial and lateral tibial loads presents several simplification in comparison to the true complex behavior of the knee subject: (i) the knee joint is modeled as a one DOF mechanism, (ii) the model properties are scaled from a healthy knee and the modifications, which may have been introduced by the knee surgery and the use of a prosthesis, are not taken into account in this study, (iii) the loads are estimated on two points on the knee rotation axis and the surface interaction of the tibial and femoral component of the prosthesis is not used. These simplifications may partly explain that the absolute load values predicted by the model for the squat movement exceed the measured values while the trends appear similar (figure 2). For gait, our simulations lead to total knee load during normal gait up to $4 * \mathrm{BW}$ (Figure 3), while literature reports maximal loads between 2 and
3*BW (Mündermann, et al., 2008). In some cases the absolute values are important. For example, they are a very relevant input for selecting the right material for orthopedic implant design.

While absolute values are essential for dimensioning and design of implants, the similarity of simulated and measured trends indicates that correct internal forces may indeed be obtained from a simulation model given correct model parameters.

The simulation suggests that the trunk sway gait actually leads to higher loads in the knee in the beginning of the stance phase while the loads in the second peak of the stance phase remains the same.

\section{CONCLUSION}

The loads obtained with the model presented in this study generally exceed measured values reported in the literature. However, the trend of the curves is similar to the in-vivo measurements. Thus, it is likely that the model is valid for prediction of the influence of different parameters (such as pathological motions, location of the prosthesis, modification of muscles insertion points) and that correct absolute values will be obtained when model parameters have been revised further. Work is currently ongoing to create a more detailed knee model for the AnyBody model repository. The current data set will prove a valuable benchmark when validating a future knee model.

\section{ACKNOWLEDGMENTS}

This work was funded by the 3D Anatomical Human EU Marie Curie Research Training Network (FP6) and the Danish National Advanced Technology Foundation.

\section{REFERENCES}

Damsgaard, M., Rasmussen, J., Christensen, S.T., Surma, E. and de Zee, M., Analysis of musculoskeletal systems in the AnyBody Modeling System. Simul. Model. Pract.Theory 14, 1059-1070. (2006)

Fregly, B.J., D'Lima, D.D., Besier, T., Lloyd, D.G., Pandy, M.G., and Banks, S.A. In Vivo Movement Data for Evaluating Musculoskeletal Model Estimates of Knee Loads. Journal of Orthopaedic Research, in preparation (for experimental data). (2010)

Lin, Y.C., Walter, J.P., Banks, S.A., Pandy, M.G., and Fregly, B.J. Simultaneous prediction of muscle and contact forces in the knee during gait. Journal of Biomechanics, in press (for implant and bone geometric models). (2010)

Kim, H.J, Fernandez, J.W., Akbarshahi, M., Walter, J.P., Fregly, B.J., and Pandy. M.G. Evaluation of predicted knee-joint muscle forces during gait using an instrumented knee implant. Journal of Orthopaedic Research 27, 1326-1331 (for implant and bone geometric models). (2009)

Klein Horsman M.D., Koopman, H.F.J.M., Van der Helm, F.C.T., Poliacu Prosé, L. and Veeger, H.E.J. Morphological muscle and joint parameters for musculoskeletal modelling of the lower extremity. Clinical Biomechanics 22, 239-247. (2007)

Mündermann, A., Dyrby, D.O., D’Lima, D.D., Colwell, C.W., Andriacchi, T.P. In vivo knee loadings characteristics during activities of daily living as measured by an instrumented total knee replacement. Journal of Orthopaedic Research 26, 1167-1172. (2008)

Rasmussen, JR, Damsgaard, M, Voigt, M. Muscle Recruitment by the min/max criterion - A comparative numerical study. Journal of Biomechanics, Vol. $34,(2001)$,

Rasmussen, J., de Zee, M., Damsgaard, M., Christensen, S.T., Marek, C., Siebertz K. A General Method for Scaling Musculo-Skeletal Models. International Symposium on Computer Simulation in Biomechanics, Cleveland, Ohio, USA. (2005)

Walker, P.S., Rovick, J.S., Robertson D.D. The effect of knee brace hinge design and placement on joint mechanics. Journal of Biomechanics 21, 965-974. (1998)

Zhao, D., Banks, S.A., D'Lima, D.D., Colwell, C.W., and Fregly, B.J. In vivo medial and lateral tibial loads during dynamic and high flexion activities. Journal of Orthopaedic Research 25, 593-602 (for medial and lateral contact force regression equations and for fluoroscopic gait data). (2007) 